PROCEEDINGS OF THE

AMERICAN MATHEMATICAL SOCIETY

Volume 138, Number 1, January 2010, Pages 309-313

S 0002-9939(09)10082-5

Article electronically published on September 4, 2009

\title{
A NOTE ON MAXIMAL AVERAGES IN THE PLANE
}

\author{
JOSÉ A. BARRIONUEVO AND LUCAS S. OLIVEIRA
}

(Communicated by Michael T. Lacey)

\begin{abstract}
Let $\mathcal{B}_{\delta}$ be the class of all $h \times \delta h$ rectangles in the plane with $h>0$ and $0<\delta<\frac{1}{2}$. The orientation of the rectangles is arbitrary. Form the maximal operator

$$
G M f(x)=\sup _{0<\delta<\frac{1}{2}} \sup _{x \in R \in \mathcal{B}_{\delta}} \frac{1}{|\log \delta| \cdot|R|} \int_{R}|f(y)| d y .
$$

Note the logarithmic term in the average. It is shown that $G M$ is a bounded
\end{abstract} maximal operator in $L^{2}\left(\mathbb{R}^{2}\right)$. The case of a fixed $\delta$ is due to Córdoba.

\section{INTRODUCTION}

For $\delta$ in $(0,1)$, let $\mathcal{B}_{\delta}$ denote the basis of all $h \times \delta h$ rectangles in $\mathbb{R}^{2}$. For a locally integrable function $f$, define the maximal operator $M_{\delta}$ by

$$
M_{\delta} f(x)=\sup _{x \in R \in \mathcal{B}_{\delta}} \frac{1}{|R|} \int_{R}|f(y)| d y .
$$

Córdoba proved in [3] that $M_{\delta}$ satisfies the following sharp $L^{2}\left(\mathbb{R}^{2}\right)$ estimate:

$$
\left\|M_{\delta}\right\| \leq C|\log \delta| .
$$

Define the following grand maximal operator:

$$
G M f(x)=\sup _{0<\delta<1 / 2} \frac{1}{|\log \delta|} M_{\delta} f(x) .
$$

We prove

Theorem 1. GM is bounded on $L^{2}\left(\mathbb{R}^{2}\right)$.

Observe that the factor $\frac{1}{|\log \delta|}$ in (1.3) is sharp. For if it is replaced by $A_{\delta}$ satisfying $\lim \sup _{\delta \rightarrow 0^{+}} A_{\delta}|\log \delta|=\infty$, then $G M$ is unbounded.

We use the following convention: If $A$ and $B$ are positive operators, $A \lesssim B$ means that there is an absolute constant $C$ such that $A f(x) \leq C B f(x)$ for all nonnegative $f$ and a.e. $x$. Also norms of vectors $\|f\|,\|T f\|$ are Lebesgue spaces $L^{2}$ norms while norms $\|S\|$ of operators are the uniform operator norm.

The authors are grateful to Michael Lacey for providing them with a number of suggestions improving an earlier version of the manuscript.

Received by the editors March 18, 2009 and, in revised form, April 20, 2009, and June 15, 2009 .

2000 Mathematics Subject Classification. Primary 42B25.

Key words and phrases. Maximal operators.

(C)2009 American Mathematical Society Reverts to public domain 28 years from publication 


\section{Remarks about the $T T^{*}$ TeChnique}

The $T T^{*}$ method is a well-known technique for proving the boundedness of a positive linear operator on $L^{2}$. It calls for one to prove the estimate

$$
T T^{*} \lesssim T+T^{*}
$$

This, with the Hilbertian fact that $\left\|T T^{*}\right\|=\|T\|^{2}=\left\|T^{*}\right\|^{2}$, shows that $\|T\| \lesssim 1$. This principle can be applied to maximal operators which are only sublinear as follows.

Let $\left\{T_{m}: m \in \mathcal{M}\right\}$ be a collection of positive linear operators, and form the maximal operator by setting

$$
T_{\max } f(x)=\sup _{m \in \mathcal{M}}\left|T_{m} f(x)\right|
$$

The inequality

$$
\left\|T_{\max } f\right\|_{L^{2}} \lesssim\|f\|_{L^{2}}
$$

is equivalent to the uniform boundedness of the family of linear maps $f \rightarrow$ $T_{\mathfrak{M}(x)} f(x)$, where $\mathfrak{M}$ is any measurable map from the underlying measure space to $\mathcal{M}$.

Suppose that one can show that

$$
T_{m} T_{n} \lesssim T_{m}+T_{n}^{*}, \quad m, n \in \mathcal{M} .
$$

Take $\mathfrak{M}$ to be a measurable map from the underlying measure space into $\mathcal{M}$. It follows that

$$
T_{\mathfrak{M}} T_{n}^{*} \lesssim T_{\mathfrak{M}}+T_{n}^{*}, \quad n \in \mathcal{M}
$$

Positivity is preserved under adjoints; hence

$$
T_{n} T_{\mathfrak{M}}^{*} \lesssim T_{\mathfrak{M}}^{*}+T_{n}, \quad n \in \mathcal{M}
$$

Then we can replace $n$ above by $\mathfrak{M}$ to deduce (2.3) for the linear operator $T_{\mathfrak{M}}$. Then one has a bound on $\left\|T_{\mathfrak{M}}\right\|$ that is independent of the choice of measurable map $\mathfrak{M}$. Hence, we deduce the boundedness of the maximal function $T_{\max }$. Arguments of this type appeared first in [5]. Other variants can be found in [1], [2], and [4].

There is an elaboration of this method here. Let $T_{m}$ be as in (2.2).

Lemma 2. Suppose that $\left\{T_{m}: m \in \mathcal{M}\right\}$ are positive uniformly bounded operators and there is an auxiliary collection of positive operators $\left\{U_{m}: m \in \mathcal{M}\right\}$ for which we can show

$$
T_{m} T_{n}^{*} \lesssim T_{\mu(m)}+T_{\mu(n)}^{*}+T_{\mu(m)} U_{n}^{*}+U_{m} T_{\mu(n)}^{*}, \quad m, n \in \mathcal{M} .
$$

Here, $\mu: \mathcal{M} \longrightarrow \mathcal{M}$ is an arbitrary fixed map. If, in addition, $U_{\max } f=$ $\sup _{m \in \mathcal{M}} U_{m} f$ is a bounded maximal operator on $L^{2}$, we can then conclude the same for $T_{\max }$.

Proof. Fix an integer $l$, and define

$$
\Lambda=\sup _{\substack{\mathcal{M}^{\prime} \subset \mathcal{M} \\ \sharp \mathcal{M}^{\prime}=l}} \sup _{\|f\|=1}\left\|\sup _{m \in \mathcal{M}^{\prime}} T_{m}(|f|)\right\| .
$$

By assumption, $\Lambda \lesssim l$, and we seek a bound on $\Lambda$ that is independent of $l$. By a limiting argument, this will finish the proof of the lemma. 
Fix $\mathcal{M}^{\prime} \subset \mathcal{M}$ with ${ }^{\sharp} \mathcal{M}^{\prime}=l$ and $\varphi \geq 0$ with $\|\varphi\|=1$ so that

$$
\Lambda<2\left\|\sup _{m \in \mathcal{M}^{\prime}} T_{m}(\varphi)\right\| \text {. }
$$

Further select a measurable map $\mathfrak{M}$ from the underlying measure space to $\mathcal{M}^{\prime}$ such that

$$
\Lambda<4\left\|T_{\mathfrak{M}}(\varphi)\right\| .
$$

We provide an estimate for the norm of the linear operator $T_{\mathfrak{M}}$.

Note that uniformity in (2.4) implies that

$$
T_{\mathfrak{M}} T_{n}^{*} \lesssim T_{\mu \circ \mathfrak{M}}+T_{\mu(n)}^{*}+T_{\mu \circ \mathfrak{M}} U_{n}^{*}+U_{\mathfrak{M}} T_{\mu(n)}^{*},
$$

where $U_{\mathfrak{M}}$ is the linear operator defined analogously to $T_{\mathfrak{M}}$. Positivity is preserved under adjoints - and we can take adjoints as all operators are linear — so we see that

$$
T_{n} T_{\mathfrak{M}}^{*} \lesssim T_{\mu \circ \mathfrak{M}}^{*}+T_{\mu(n)}+U_{n} T_{\mu \circ \mathfrak{M}}^{*}+T_{\mu(n)} U_{\mathfrak{M}}^{*}
$$

This is a uniform inequality; hence

$$
T_{\mathfrak{M}} T_{\mathfrak{M}}^{*} \lesssim T_{\mu \circ \mathfrak{M}}^{*}+T_{\mu \circ \mathfrak{M}}+U_{\mathfrak{M}} T_{\mu \circ \mathfrak{M}}^{*}+T_{\mu \circ \mathfrak{M}} U_{\mathfrak{M}}^{*}
$$

The operator norm of the term on the left is, by construction, comparable to $\Lambda^{2}$. Note that $\mu \circ \mathfrak{M}$ is a map from the underlying measure space to $\mu\left(\mathcal{M}^{\prime}\right)$, which is a subset of $\mathcal{M}$ of cardinality $l$. Thus, each term on the right, by selection of an extremal $\mathcal{M}^{\prime}$, has operator norm dominated by a constant times $\Lambda\left(1+\left\|U_{\max }\right\|\right)$. So we have a bound on $\Lambda$ that is independent of $l$, and the proof is finished.

\section{Principal line of ARGUMent}

Let $\mathcal{M}=(0, \infty) \times\left[0, \frac{1}{10}\right] \times\left(\delta_{0}, \frac{1}{2}\right)$. For $m=(h, \theta, \delta) \in \mathcal{M}$, let $R_{m}$ be the $h \times \delta h$ rectangle centered at the origin, with longest side having an angle $\theta$ with the $x$-axis. Define

$$
\begin{aligned}
T_{m} f(x) & =\frac{1}{|\log \delta|\left|R_{m}\right|} \int_{R_{m}} f(x-y) d y \\
\operatorname{GMf}(x) & =\sup _{m \in \mathcal{M}}\left|T_{m} f(x)\right| .
\end{aligned}
$$

We prove

$$
T_{m} T_{n} \lesssim T_{m^{\prime}}+T_{n^{\prime}}+T_{m^{\prime}} W_{n}+W_{m} T_{n^{\prime}}
$$

where for $m=(h, \theta, \delta) \in \mathcal{M}$ we set $m^{\prime}=(2 h, \theta, \delta)$. We now define the operators $W_{m}$ :

$$
\begin{aligned}
W_{m} f(x) & =\frac{1}{2|\log \delta|} \sum_{j=0}^{2|\log \delta|} H_{m, j} f(x), \\
H_{m, j} f(x) & =\frac{1}{\delta h 2^{j+1}} \int_{-\delta h 2^{j}}^{\delta h 2^{j}} f\left(x+t \overrightarrow{\mathfrak{e}}_{2}\right) d t,
\end{aligned}
$$

where $\overrightarrow{\mathfrak{e}}_{2}=(0,1)$ is the second coordinate direction in the plane. By construction, the rectangles we deal with essentially point in the first coordinate direction in the plane. If $M^{2}$ denotes the one-dimensional maximal operator computed in the second coordinate, then we have $W_{m} f \lesssim M^{2} f$. In view of (2.4), this will prove the main theorem. 
Turning to the proof of (3.1), we set $m=(h, \theta, \delta)$ and $n=\left(h^{\prime}, \theta^{\prime}, \delta^{\prime}\right)$. Let us assume that $h>h^{\prime}$, which assumption we return to below.

Lemma 3. For $f \geq 0$ we have the pointwise inequality

$$
T_{m} T_{n} f(x) \lesssim \frac{1}{|\log \delta| \cdot\left|\log \delta^{\prime}\right| \cdot|R|} \int_{R} f(x-y) d y,
$$

where $R$ is the rectangle centered at the origin, of dimensions $2 h \times 2 w$, where

$$
w=\max \left\{\delta h, \delta^{\prime} h^{\prime}, h^{\prime} \sin \left|\theta-\theta^{\prime}\right|\right\},
$$

and the longest side of $R$ is $2 h$, which forms an angle $\theta$ with the $x$-axis.

Proof. Since $T_{m}$ has kernel

$$
K_{m}(x, y)=\frac{1}{|\log \delta| \cdot\left|R_{m}\right|} \mathbf{1}_{R_{m}}(x-y),
$$

the kernel of $T_{m} T_{n}$ is

$$
\begin{aligned}
K_{m, n}(x, y) & =\frac{1}{|\log \delta| \cdot\left|\log \delta^{\prime}\right| \cdot\left|R_{m}\right| \cdot\left|R_{n}\right|} \int \mathbf{1}_{R_{m}}(x-s) \mathbf{1}_{R_{n}}(s-y) d s \\
& =\frac{\left|\left(R_{m}+x\right) \cap\left(R_{n}+y\right)\right|}{|\log \delta| \cdot\left|\log \delta^{\prime}\right| \cdot\left|R_{m}\right| \cdot\left|R_{n}\right|}
\end{aligned}
$$

First note that this implies that

$\operatorname{supp} K_{m, n} \subset\left\{(x, y) \in \mathbb{R}^{2} \times \mathbb{R}^{2}: x-y \in R_{m}+R_{n}\right\} \subset\left\{(x, y) \in \mathbb{R}^{2} \times \mathbb{R}^{2}: x-y \in R\right\}$ where $R$ is the rectangle described in the statement of the lemma. Second, if $(x, y) \in \operatorname{supp} K_{m, n}$, the above implies

$$
\begin{aligned}
K_{m, n}(x, y) & \lesssim \frac{\left|R_{m} \cap R_{n}\right|}{|\log \delta| \cdot\left|\log \delta^{\prime}\right| \cdot\left|R_{m}\right| \cdot\left|R_{n}\right|} \\
& \lesssim \frac{1}{|\log \delta| \cdot\left|\log \delta^{\prime}\right| \cdot|R|},
\end{aligned}
$$

and the lemma follows from these two observations.

The argument for (3.1) depends upon the value of $w$. In the case that $w=\delta h$, it follows that $R$ is a $2 h \times 2 \delta h$ rectangle; hence

$$
T_{m} T_{n} \lesssim\left|\log \delta^{\prime}\right|^{-1} T_{m^{\prime}} \lesssim T_{m^{\prime}}
$$

In the case $w=\max \left\{\delta^{\prime} h^{\prime}, h^{\prime} \sin \left|\theta-\theta^{\prime}\right|\right\}$, there is a choice of integer $j=$ $1, \ldots, 2\left[\log \delta^{\prime}\right]$ such that $2^{j-1} \delta^{\prime} h^{\prime} \leq w<2^{j} \delta^{\prime} h^{\prime}$, whence,

$$
\begin{aligned}
T_{m} T_{n} & \lesssim\left|\log \delta^{\prime}\right|^{-1} T_{m} H_{n, j} \\
& \lesssim\left|\log \delta^{\prime}\right|^{-1} T_{m^{\prime}} \sum_{j=1}^{2\left[\left|\log \delta^{\prime}\right|\right]} H_{n, j} \\
& \lesssim T_{m^{\prime}} W_{n} .
\end{aligned}
$$

We have passed to the larger sum in the middle step in order to get an estimate that depends solely on $n$. To recap, in the case that $h>h^{\prime}$, we have shown

$$
T_{m} T_{n} \lesssim T_{m^{\prime}}+T_{m^{\prime}} W_{n},
$$

which proves 3.1 in this case. 
Finally, if $h^{\prime}>h$, the operators $T_{m}$ and $T_{n}$ commute, so that the argument just given supplies us with the estimate

$$
T_{m} T_{n} \lesssim T_{n^{\prime}}+T_{n^{\prime}} W_{m}
$$

Summing this inequality and (3.2) proves (3.1) as stated.

\section{REFERENCES}

1. J. Barrionuevo, Estimates for some Kakeya-type maximal operators, Trans. Amer. Math. Soc. 335 (1993), 667-682. MR1150012 (93f:42038)

2. — A note on the Kakeya maximal operator, Math. Res. Lett. 3, no. 1 (1996), 61-65. MR.1393383 (98k:42023)

3. A. Córdoba, The Kakeya maximal function and the spherical summation multipliers, Amer. J. Math. 99 (1977), 1-22. MR0447949 (56:6259)

4. A. Nevo, Harmonic analysis and pointwise ergodic theorems for noncommuting transformations, J. Amer. Math. Soc. 7 (1994), 875-902. MR1266737 (95h:22006)

5. E. M. Stein, On the maximal ergodic theorem, Proc. Nat. Acad. Sci. USA 47 (1961), 18941897. MR0131517 (24:A1367)

Departamento de Matemática, Universidade Federal Rio Grande do Sul, Av. Bento Gonçalves 9500, 91509-900 Porto Alegre, RS, Brasil

E-mail address: josea@mat.ufrgs.br

Departamento de Matemática, Universidade Federal Rio Grande do Sul, Av. Bento Gonçalves 9500, 91509-900 Porto Alegre, RS, Brasil

E-mail address: lucas_gnomo@hotmail.com 